\title{
Adipocytes modulate vascular smooth muscle cells migration potential through their secretions ${ }^{*}$
}

\author{
Souhad El Akoum ${ }^{1,2}$, Isabelle Cloutier ${ }^{1}$, Jean-François Tanguay ${ }^{1,2 \#}$ \\ ${ }^{1}$ Montreal Heart Institute, Montréal, Canada; ${ }^{\#}$ Corresponding Author: jean-francois.tanguay@,icm-mhi.org \\ ${ }^{2}$ Département de Sciences Biomédicales, Faculté de Médecine, Université de Montréal, Montréal, Canada
}

Received 3 May 2013; revised 3 June 2013; accepted 12 June 2013

Copyright (C) 2013 Souhad El Akoum et al. This is an open access article distributed under the Creative Commons Attribution License, which permits unrestricted use, distribution, and reproduction in any medium, provided the original work is properly cited.

\begin{abstract}
Impairment of vascular smooth muscle cells (VSMC) is recognized as a predisposition factor for atherosclerosis development. We hypothesize that the metabolic syndrome has a direct impact on VSMC migration and phenotypic switching, which may increase the incidence of atherosclerotic events. Aortic VSMC were extracted from 10 weeks old C57BL6 mice and incubated for $\mathbf{2 4} \mathrm{hr}$ in adipocytes conditioned cell culture medium. Adipocytes were extracted from diabetic C57BL6 male mice fed with either a vegetal or an animal High-Fat-Diet (HFD) for 20 weeks. Migration of VSMC in response to conditioned media stimulations was significantly modulated compared to control. The most extended effects on VSMC were triggered by adipocytes from mice fed with animal HFD. These effects were concurrent with increased leptin concentrations and decreased adiponectin levels in conditioned media. A significant up-regulation of CD36 mRNA level was found in VSMC treated with adipocytes from HFD-fed mice. In conclusion, we have shown that the development of adipocyte-induced VSMC alterations is linked to diet fatty acid composition and the degree of metabolic alterations. The modulation of adipokine secretions in the adipose tissue that is linked to metabolic alterations may alter the physiology of VSMC and thus accelerate the development of metabolic-related vascular diseases.
\end{abstract}

Keywords: Adipocytes; Atherosclerosis; Type 2

\footnotetext{
${ }^{*}$ Funding: This study was financed by a grant from the foundation des maladies du Coeur du Québec and the Montreal Heart Institute foundation.

Competing of interest: Neither of the authors has any potential benefits or conflicts of interest to disclose.
}

\section{Diabetes; Vascular Smooth Muscle Cells; Migration}

\section{INTRODUCTION}

Obesity and type 2 diabetes (T2D) are recognized as predisposition factors for atherosclerosis development. Endothelial cells (EC) and vascular smooth muscle cells (VSMC) are sensitive to obesity-linked increased fatty acids (FA) and T2D-linked hyperglycæmia that triggers vascular alterations and leads to atherogenesis [1-3]. A lack in insulin sensitivity lowers intracellular glucose availability forcing arterial cells to use FA as an alternative energy source. Thus, in atheroprone regions susceptible to plaque formation, EC and VSMC are subjected to metabolic modifications that lead to the accumulation of oxidized LDL (oxLDL) within the intima [4] and the progression of vascular diseases.

At the early stage of atherogenesis, the oxLDL-exposed endothelium becomes activated and up-regulates the expression of adhesion molecules (ICAM, VCAM), thereby allowing monocyte recruitment and initiation of the inflammatory process [4]. For their part, VSMC undergo "phenotypic switching", which is characterized by decreased contractibility and increased proliferation and migration toward the intima. These processes then contribute to intima thickness and atherosclerotic plaque formation $[5,6]$. With time, VSMC may undergo cholesterol-induced trans-differentiation into foam cells [7]. These cells have a macrophage like phenotype and express increased levels of the oxLDL scavenger receptor CD36, which favors lipid accumulation and contributes to the atherogenic process [8].

The specific factors implicated in VSMC alterations linked to obesity and T2D remain incompletely understood. However, the migration process of these cells seems to play a pivotal role in the development of atherosclerosis and its complications. The secretions of adipokines from the adipose tissue, which regulate various sets of metabolic and physiologic processes, could 
not be discarded $[9,10]$. Indeed, little is known about the effect of these adipokines on the VSMC migration process. Thus, since obesity-increased FA is an important inducer of metabolic disorders and vascular pathogenesis, and regulates adipose tissue activity, we hypothesized that adipocytes through adipokine secretions play an important role in VSMC alterations leading to atherosclerosis.

Therefore, the present study is aimed at determining the effect of factors secreted by adipocytes isolated from mice fed with High-Fat-Diet (HFD) on VSMC migration. We focused our investigation on isolated adipocytes to exclude the impact of other cell types found in the adipose tissue such as macrophages.

\section{MATERIALS AND METHODS}

\subsection{Experimental Protocol}

Adipocytes were obtained from C57BL/6J male mice fed on a HFD for 20 weeks [11]. Mice were fed one of two low cholesterol HFD (34.9\% fat, $26.3 \%$ carbohydrate, cholesterol $<0.03 \%$; ResearchDiet): a vegetal HFD (VD) composed of soy and cotton oil and an animal HFD (AD) composed of lard. The common standard diet (SD) was used as control (6\% fat, $57 \%$ carbohydrate; HarlanTeklad). The animal protocol was approved by the Animal Care and Use Committee of the Montreal Heart Institute.

\subsection{Adipocyte Culture}

Mature adipocytes were isolated from visceral-abdominal adipose tissue of each mouse at sacrifice. Briefly, adipose tissue was finely minced and enzymatically digested in Dulbecco's-Modified-Eagle-Medium (DMEM) containing $2 \mathrm{mg} / \mathrm{ml}$ type I collagenase $\left(37^{\circ} \mathrm{C} ; 40 \mathrm{~min}\right)$. Samples were then centrifuged $(300 \times \mathrm{g} ; 7 \mathrm{~min})$ and the top white supernatant passed through $100 \mu \mathrm{m}$ filter and washed twice in DMEM.

Adipocytes $\left(10^{4}\right.$ cells $\left./ \mathrm{ml}\right)$ were maintained in serum free low glucose DMEM supplemented or not with insulin $(100 \mu \mathrm{U} / \mathrm{ml})$, glucose $(25 \mathrm{mM})$ or insulin + glucose for $24 \mathrm{hr}$. Adipocytes supernatants (AdS) were collected and immediately stored at $-20^{\circ} \mathrm{C}$. At the moment of experimentation samples were thawed and added in VSMC cultures.

To simplify annotations, supernatants from unstimulated adipocytes were noted as AdS-U, supernatants from insulin-stimulated adipocytes as AdS-I, supernatant from glucose-stimulated adipocytes as AdS-G and supernatant from (insulin + glucose)-stimulated adipocytes as AdS-IG.

\subsection{VSMC Culture}

VSMC were isolated from 10 weeks old C57BL/6J mice as previously described [12]. Briefly, dissected aor- tas were discarded of its adventitia, cut in $2 \mathrm{~mm}$ square pieces and incubated in $1.5 \mathrm{mg} / \mathrm{ml}$ collagenase type II solution $\left(37^{\circ} \mathrm{C} ; 5 \% \mathrm{CO}_{2} ; 5 \mathrm{hr}\right)$. Dissociated cells were suspended in $5 \mathrm{ml}$ of DMEM and centrifuged at $300 \times \mathrm{g}$ for $5 \mathrm{~min}$. The pellet was resuspended in $700 \mu \mathrm{LMEM}$ supplemented with $10 \%$ serum and transferred to a single well of a 48-well plate and left untreated for 5 days. VSMC purity was assessed by confocal microscopy upon 4 amplification passages [12] before treatment with AdS. To separate the effects of insulin and glucose from the effects of secreted factors from adipocytes on VSMC in AdS, insulin and glucose stimulations on VSMC were used as controls for each experiment. Each condition was tested in duplicate and expressed as percentage compared to control.

\subsection{Cell Migration Assay}

Confluent VSMC were serum starved overnight before a $24 \mathrm{hr}$ treatment with AdS or appropriate controls. Cells $\left(5 \times 10^{3}\right)$ were loaded into the upper chamber of a $24-$ transwell plate with an $8 \mu \mathrm{m}$ pore membrane. Fibroblast growth factor (bFGF), which was used as the chemoattractant, was added into the lower chambers at $10 \mathrm{ng} /$ $\mathrm{ml}$. Following $5 \mathrm{~h}$ of incubation $\left(37^{\circ} \mathrm{C} ; 5 \% \mathrm{CO}_{2}\right)$, the membrane was fixed with paraformaldehyde, stained with 4',6'-diamidino-2-phenylindole and scanned on a microscope. The number of migrated cells was counted in five random fields for each sample.

\subsection{Characterization of VSMC}

Cells were cultured and allowed to grow near confluence on $8 \mathrm{~mm}$ glass lamella. Following two washes in DMEM, cells were fixed in $4 \%$ paraformaldehyde and permeabilized with $0.1 \%$ Triton X-100. The cells were then washed and subsequently blocked for $1 \mathrm{hr}$ in $2 \%$ normal goat serum. After washing with PBS BSA1\%, cells were incubated with an anti- $\alpha$ smooth muscle actin antibody (NeoMarker, USA) over night at $4^{\circ} \mathrm{C}$. After incubation, the cells were washed twice in PBS BSA 1\% and further incubated with an affinity purified Alexa 488conjugated anti-rabbit antibody (Invitrogen, USA). Fluorescent specimens were visualized under a microscope and photographed.

FA incorporation in AdS-treated VSMC was evaluated by Oil-Red-O staining. Cells were fixed in $10 \%$ formalin for 15 minutes. $60 \%$ isopropanol was then added before completely drying wells. A stock of $0.5 \%$ ORO staining solution was prepared in $60 \%$ triethyl phosphate (TEP, Sigma) and filtered. A working solution was prepared by mixing $30 \mathrm{ml}$ of ORO stock solution with $50 \mathrm{ml}$ of PBS, filtered through a $1 \mu \mathrm{m}$ filter and added to the dried wells. Following a 1-hour incubation period, the ORO solution was removed and cells were washed in PBS prior to pic- 
ture acquisition using a light microscope equipped with a video camera.

\subsection{Quantification of mRNA Level}

Total RNA was isolated using Qiazol reagent according to the manufacture's instructions (Qiagen, Toronto, ON, Canada). Single-strand cDNA was synthesized according to the procedure in the iScript cDNA Synthesis Kit manual (Bio-Rad Laboratories, Montreal, QC, Canada). Q-PCR reactions were carried out using the Brilliant-II SYBR ${ }^{\circledR}$ Green Master-Mix (Stratagene, Mississauga, ON, Canada) and specific primers for CD36 scavenger receptor (Fwd: 5'-GCC-AAG-CTA-TTG-CGA-CATGA-3'; Rev: 5'-AAG-GCA-TTG-GCT-GGA-AGA-AC3') and insulin receptor (Fwd: 5'-CAG-AGA-AGG-TCTCTC-GGA-CT-3'; Rev: 5'-TGG-AGA-GGT-AGA-TGAGCC-GC-3'). The mRNA levels were normalized to Cyclophilin-A expression levels (Fwd: 5'-CCG-ATGACG-AGC-CCT-TGG-3'; Rev: 5'-GCC-GCC-AGTGCC-ATT-ATG-3'). The targeted and reference genes were amplified in duplicates using the Mx3000P Q-PCR System (Stratagene). The relative quantification of target genes was determined using the MxProTM Q-PCR software version 3.00 (Strategene) as previously described [12].

\subsection{Biochemical Assay}

Adiponectin and leptin concentrations within AdS were measured using a mouse ELISA kit according to the manufacturer's protocol (ALPCO). Prior to analysis, samples were thawed on ice and rapidly used.

\subsection{Statistical Analysis}

All statistical analyses were performed separately for males and females. Data are presented as mean \pm stan- dard deviation for continuous variables.

Repeated measures analysis of variance (ANOVA) models were used to compare adiponectin and leptin levels within supernatants from adipocytes between groups ( $\mathrm{SD}, \mathrm{VD}$ and $\mathrm{AD}$ groups). Cell migration data and mRNA levels were compared between groups (SD, VD and AD groups) using an ANOVA model.

In addition, the relationships among adiponectin and leptin levels, and mRNA levels and migration parameters were investigated using Pearson or Spearman correlations according to the nature of the distribution data.

All analyses were done using SAS version 9.1 (SAS Institute Inc., Cary, NC, USA) and data $\leq 0.05$ were considered significance.

\section{RESULTS}

HFD-triggered obesity in corresponding mouse groups correlated with increased leptin levels in systemic circulation (Table 1) [11]. The development of T2D in HFD groups within male mice was also linked to decreased adiponectin levels in sera. In the $\mathrm{AD}$ group, mice also demonstrated hyperinsulinemia. The blood profile of adipokines was diet-dependant, as HFD-fed mice showed decreased adiponectin and increased leptin mRNA levels within the abdominal visceral white adipose tissue, in comparison to SD-fed mice. To characterize the effect of these modulations on VSMC, abdominal adipocytes were isolated from each animal group and used to condition culture media.

\subsection{Biochemical Characteristics}

To determine whether the differential effects of the AdS subtypes were attributable to variation in production of adipokines, we examined their leptin and adiponectin contents. These two adipokines are known for their important role in cellular glucose and lipid homeostasis.

Table 1. In vivo mice parameter after 20 weeks of diet. Weight gain, glycemic parameters and adipokines secretion profile evaluated in fasted sera mice. mRNA levels of leptin and adiponectin in visceral abdominal adipose tissue were also evaluated at the sacrifice. ${ }^{*} \mathrm{P}<0.05,{ }^{* *} \mathrm{P}<0.01,{ }^{* * *} \mathrm{P}<0.001$ vs. SD; ${ }^{\#} \mathrm{P}<0.05,{ }^{\# \#} \mathrm{P}<0.01$ vs. VD.

\begin{tabular}{|c|c|c|c|}
\hline & \multicolumn{3}{|c|}{ Diet groups } \\
\hline & $\mathrm{SD}$ & VD & $\mathrm{AD}$ \\
\hline Weight gain (g) & $11.8 \pm 0.9$ & $28.1 \pm 1.3^{* * *}$ & $23.9 \pm 1.6^{* * *}$ \\
\hline Fasting glycæmia (mM) & $4.0 \pm 0.2$ & $9.0 \pm 0.6^{* * *}$ & $8.3 \pm 0.6^{* * *}$ \\
\hline Fasting insulinæmia $(\mu \mathrm{g} / \mathrm{ml})$ & $0.3 \pm 0.1$ & $0.4 \pm 0.1$ & $0.7 \pm 0.1^{* * \#}$ \\
\hline Fasting leptin level $(\mu \mathrm{g} / \mathrm{ml})$ & $0.06 \pm 0.013$ & $0.58 \pm 0.05^{* * *}$ & $0.8 \pm 0.07^{* * * \# \#}$ \\
\hline Fasting adiponectin level $(\mu \mathrm{g} / \mathrm{ml})$ & $1.97 \pm 0.15$ & $1.40 \pm 0.12^{* *}$ & $1.24 \pm 0.15^{* * *}$ \\
\hline Fasting FA level $(\boldsymbol{\mu M})$ & $0.51 \pm 0.12$ & $1.13 \pm 0.04^{*}$ & $1.18 \pm 0.22^{*}$ \\
\hline Leptin mRNA level in adipose tissue & $0.78 \pm 0.18$ & $3.93 \pm 0.26^{* * *}$ & $5.39 \pm 0.64^{* * *}$ \\
\hline Adiponectin mRNA level in adipose tissue & $1.24 \pm 0.08$ & $0.53 \pm 0.06^{* * *}$ & $0.61 \pm 0.06^{* * *}$ \\
\hline
\end{tabular}


Their inverse interactions are linked to metabolic disorders and vascular alterations.

Leptinemia was increased by 4 fold in AdS-U from HFD groups, as compared to the SD group (Table 2). Hyper-leptinemia was further increased in response to insulin and/or glucose stimulations in HFD groups, compared to unstimulated cells where maximum levels were reached with AdS-G.

On the other hand, adiponectin levels were decreased as expected in AdS-U of adipocytes from HFD-fed mice, in comparison to the SD group (Table 2). In the SD group, adiponectin concentrations were increased by $30 \%$ in AdS-I, 70\% in AdS-IG, and $96 \%$ in AdS-G, as compared to AdS-U. The effect of insulin adiponectin secretions was not significant in $\mathrm{AD}$ and $\mathrm{VD}$ group, compared to unstimulated cells. However, it was increased by the addition of glucose (AdS-IG: $140 \%$ for VD and $127 \%$ for AD) or glucose alone (AdS-G: $350 \%$ for $\mathrm{VD}$ and $222 \%$ for AD), compared to unstimulated fat cells. Despite these differences, adiponectin concentrations in HFD-derived AdS remained 30\% to $70 \%$ higher in the corresponding controls from the SD group (Table 2).

\subsection{Effects of AdS on VSMC Migration}

The migration process of VSMC is critical for vasculogenesis and vascular repair. Insulin and glucose treatments significantly increased VSMC migration by 10 to $33 \%$, compared to untreated cells (Figure 1(B)). To characterize the impact of AdS on VSMC physiology, we evaluated VSMC migration capabilities after $24 \mathrm{hr}$ of treatment with AdS. All AdS from SD-derived adipocytes reduced migration of VSMC; albeit to a lesser extend in the case of AdS-G (Figure 1(A)). In contrast, all AdS from HFD-fed mice showed a strong pro-migratory effect on VSMC (65\% - 70\% increase). This diet-induced effect was more prominent in HFD groups with AdS-I (200\%), AdS-IG (230\%) and AdS-G (260\%), compared to AdS-U.

The impact of AdS on VSMC migration was also reflected by their cytoskeleton arrangement. While freshly extracted VSMC showed a twisted form with parallel $\alpha$-actin filaments [12], this process was absent in AdS-U from SD mice, while light disorganization of the actin filaments was noted (Figure S1(A)). Conversely, in the AdS-U-VD group, VSMC showed disorganized parallel actin filament structures with some migratory extensions (Figure S1(B)), while supernatants from AD groups illustrated increased fibers' disorder (Figure S1(C)). These effects were not associated with parallel $\alpha$-actin filaments and microscopic snapshots showed blurring and dot staining, reporting most probably actin polymerization that lead to extension of the leading edge.

\subsection{Insulin Receptor Gene Modulation}

Since the metabolism of VSMC depends upon glucose, we evaluated the gene expression profile of InsR, which is responsible for glucose uptake. In control VSMC, only insulin and glucose stimulations significantly reduced InsR expression levels $(0.55 \pm 0.02)$, compared to un-

Table 2. Adiponectin and leptin levels in adipocytes' supernatants (AdS). Adiponectin and leptin levels were evaluated in AdS of untreated (AdS-U), insulin-treated (AdS-I), glucose-treated (AdS-G) and insulin and glucose treated (AdS-IG) mature adipocytes extracted from visceral abdominal adipose pad of each mice group. Treatments were maintained for $24 \mathrm{~h}$. For a same diet: ${ }^{*} \mathrm{P}<0.05$ vs AdS-U; ${ }^{* *} \mathrm{P}<0.05$ vs. AdS-I; ${ }^{* * *} \mathrm{P}<0.05$ vs. AdS-IG. For a same stimulation: ${ }^{\dagger} \mathrm{P}<0.05$ vs. SD; ${ }^{\dagger \dagger} \mathrm{P}<0.05$ vs. VD.

\begin{tabular}{|c|c|c|c|}
\hline Diet groups & Adipocytes' supernatants & Adiponectin level $(\mathrm{ng} / \mathrm{ml}) \pm \mathrm{SEM}$ & Leptin level $(\mathrm{ng} / \mathrm{ml}) \pm \mathrm{SEM}$ \\
\hline \multirow{4}{*}{ SD } & AdS-U & $2.75 \pm 0.46$ & $0.33 \pm 0.03$ \\
\hline & AdS-I & $3.61 \pm 0.21^{*}$ & $0.47 \pm 0.04$ \\
\hline & AdS-G & $4.69 \pm 0.04^{* * *}$ & $0.55 \pm 0.03^{*}$ \\
\hline & AdS-IG & $5.40 \pm 0.51^{* * *}$ & $0.77 \pm 0.05^{* * * * *}$ \\
\hline \multirow{4}{*}{ VD } & AdS-U & $0.75 \pm 0.11^{\dagger}$ & $1.20 \pm 0.09^{\dagger}$ \\
\hline & AdS-I & $0.82 \pm 0.27^{\dagger}$ & $1.78 \pm 0.05^{* \dagger}$ \\
\hline & AdS-G & $1.79 \pm 0.29^{* * * \dagger}$ & $2.25 \pm 0.16^{* * * \dagger}$ \\
\hline & AdS-IG & $3.36 \pm 0.62^{* * * * * * \dagger}$ & $2.74 \pm 0.09^{* * * * * * \dagger}$ \\
\hline \multirow{4}{*}{$\mathrm{AD}$} & AdS-U & $1.00 \pm 0.22^{\dagger}$ & $1.42 \pm 0.09^{\dagger \dagger \dagger}$ \\
\hline & AdS-I & $1.43 \pm 0.14^{\dagger \dagger \dagger}$ & $1.81 \pm 0.03^{* \dagger}$ \\
\hline & AdS-G & $2.27 \pm 0.39^{* * * \dagger}$ & $2.18 \pm 0.07^{* * * \dagger}$ \\
\hline & AdS-IG & $3.22 \pm 0.38^{* * * * * * \dagger}$ & $2.65 \pm 0.04^{* * * * * * \dagger}$ \\
\hline
\end{tabular}




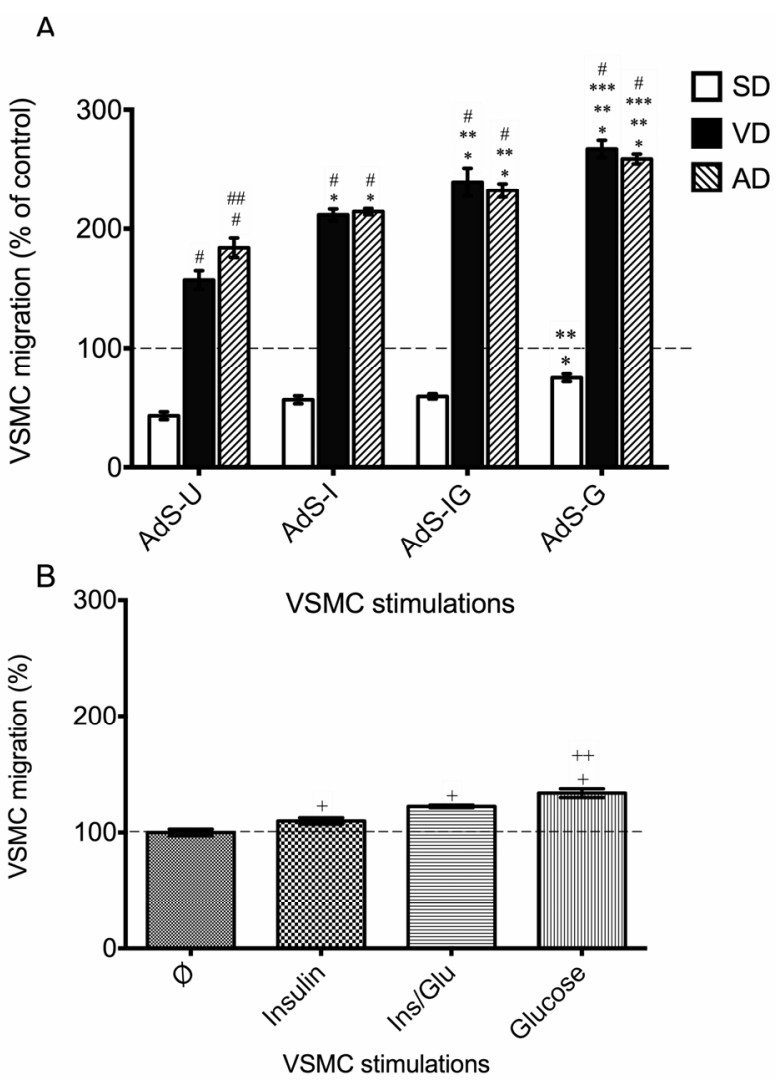

Figure 1. (A) VSMC migration rate after $24 \mathrm{hr}$ of treatment with AdS of male mice. These results are expressed as percentage of proliferation rate of unstimulated cells; (B) Incubation of VSMC with insulin or glucose for 24 hours was used as control compared to unstimulated cells. For a same diet: ${ }^{*} \mathrm{P}<0.05$ vs AdS-U; ${ }^{* *} \mathrm{P}<0.05$ vs. AdS-I; ${ }^{* * *} \mathrm{P}<0.05$ vs. AdS-IG. For a same stimulation: ${ }^{\#} \mathrm{P}<0.05$ vs. SD; ${ }^{\# \#} \mathrm{P}<0.05$ vs. VD. In control treatments: ${ }^{+} \mathrm{P}<0.05$ vs unstimulated cells $(\varnothing) ;{ }^{++} \mathrm{P}<0.05$ vs insulin stimulated cells.

stimulated $(1.01 \pm 0.11)$ and insulin $(0.85 \pm 0.11)$ or glucose $(1.22 \pm 0.25)$ stimulated cells (Figure 2(B)). In AdS-treated cells, unstimulated adipocytes did not induce any significant modulation of InsR mRNA in VSMC compared to control (Figure 2(A)). Stimulation of adipocytes from the SD group with insulin or glucose significantly decreased InsR expression levels by 30 and $75 \%$ respectively, compared to unstimulated cells. In contrast, adipocytes from $\mathrm{VD}$ and $\mathrm{AD}$ groups showed no effect on InsR gene expression in VSMC compared to unstimulated condition. Moreover, insulin + glucose stimulation was without any effect on InsR gene expression in SD groups, compared to unstimulated adipocytes. Furthermore, while adipocytes from $\mathrm{AD}$ groups slightly increased InsR mRNA levels in VSMC compared to unstimulated cells, those from VD groups markedly increased InsR levels in VSMC $(1.25 \pm 0.11)$ in response to insulin + glucose stimulation compared to the other culture conditions and to SD derived AdS.

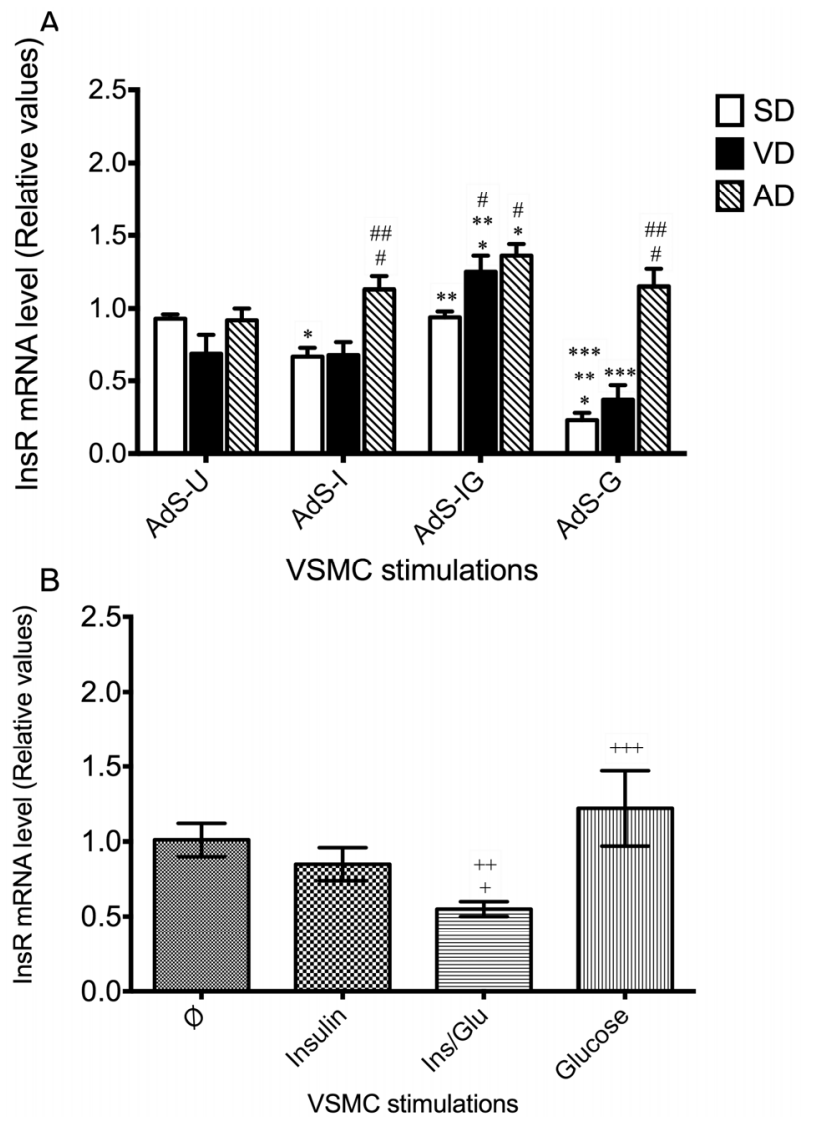

Figure 2. (A) InsR mRNA expression level in VSMC is upregulated with AdS stimulation extracted from $\mathrm{AD}$-fed group; (B) The basic InsR VSMC expression level (Ø) is reported as $100 \%$ expression gene level. Insulin + glucose stimulation decrease InsR mRNA level without any significant modulation of this parameter after insulin or glucose VSMC treatment (B). For a same diet group: ${ }^{*} \mathrm{P}<0.05$ vs AdS-U; ${ }^{* *} \mathrm{P}<0.05$ vs. AdS-I; ${ }^{* * *} \mathrm{P}<0.05$ vs. AdS-IG. For a same stimulation: ${ }^{\#} \mathrm{P}<$ 0.05 vs. SD; ${ }^{\#} \mathrm{P}<0.05$ vs. VD. In control treatments: ${ }^{+} \mathrm{P}<0.05$ vs unstimulated cells $(\varnothing),{ }^{++} \mathrm{P}<0.01$ vs insulin-stimulated cells, ${ }^{+++} \mathrm{P}<0.01$ vs insulin + glucose $(\mathrm{Ins} / \mathrm{Glu})$-stimulated cells

\subsection{VSMC Fatty Acids Accumulation Formation}

The impact of AdS on VSMC physiology could also be linked to FA released by adipocytes. Modulation of FA receptors may be an indicator of their implication in VSMC alterations. We evaluated CD36 mRNA expression levels in VSMC, a scavenger receptor recognized for its role in the atherogenesis process [8]. Insulin stimulation increased CD36 mRNA expression levels by 2 fold in VSMC, compared to unstimulated and glucose stimulated cells (Figure 3(B)). In addition, glucose alone increased CD36 mRNA levels by 30\% (Figure 3(B)). In AdS treated cells, when VSMC were exposed to AdS-U from HFD groups, CD36 mRNA levels were significantly enhanced $(0.48 \pm 0.05$ for $\mathrm{VD} ; 0.88 \pm 0.55$ for $\mathrm{AD})$, compared to SD groups $(0.02 \pm 0.13)$ (Figure 

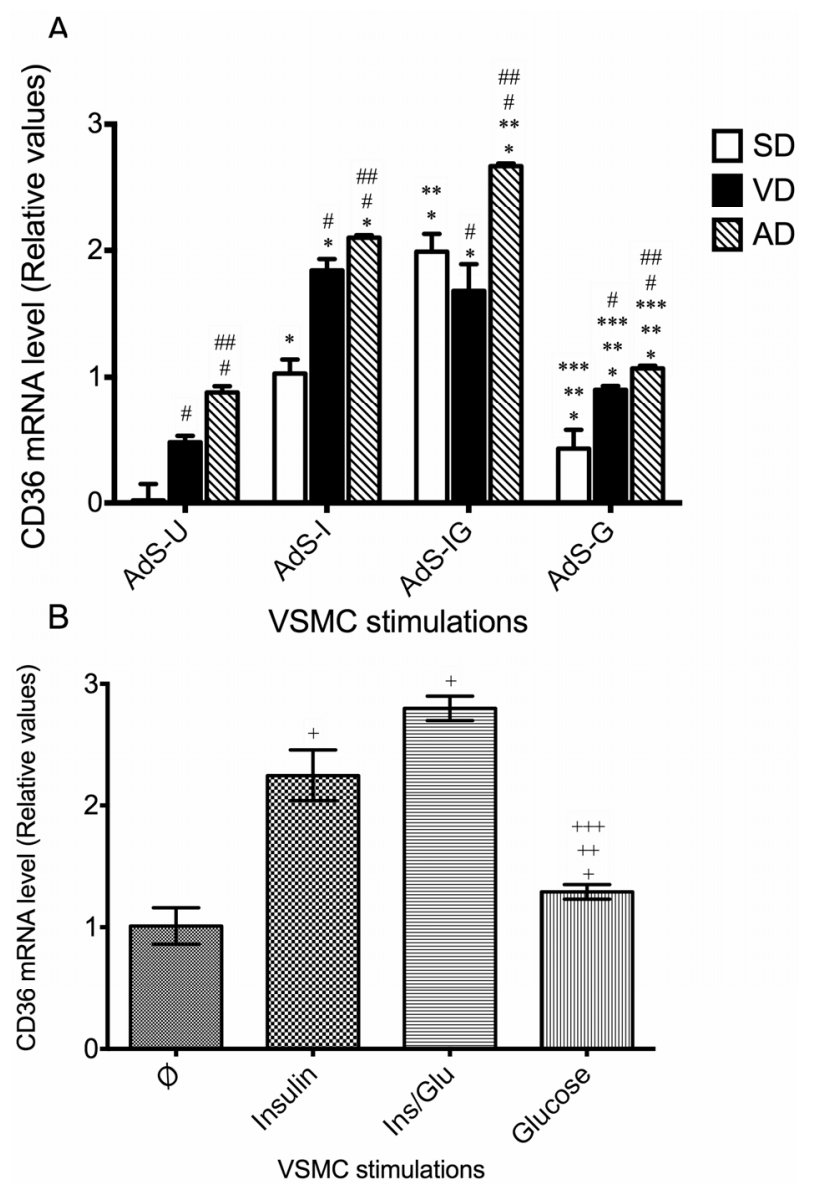

Figure 3. (A) CD36 scavenger receptor mRNA expression level in AdS-treated VSMC in different mouse groups is modulated depending on the type of treatment; (B) Incubation of VSMC with insulin or glucose for 24 hours was used as control compared to unstimulated cells. For a same diet: ${ }^{*} \mathrm{P}<0.05$ vs AdS-U; ${ }^{* *} \mathrm{P}<0.05$ vs. AdS-I; ${ }^{* * *} \mathrm{P}<0.05$ vs. AdS-IG. For a same stimulation: ${ }^{\#} \mathrm{P}<0.05$ vs. $\mathrm{SD} ;{ }^{\# \#} \mathrm{P}<0.05$ vs. VD. In control treatments: ${ }^{+} \mathrm{P}<0.05$ vs unstimulated cells $(\varnothing) ;{ }^{++} \mathrm{P}<0.05$ vs insulin stimulated cells; ${ }^{++} \mathrm{P}<0.05$ vs insulin/glucose stimulated cells.

3(A)). AdS-I of all diet groups increased CD36 mRNA expression levels in VSMC with still higher levels in VD $(1.84 \pm 0.09)$ and $\mathrm{AD}(2.10 \pm 0.02)$ groups, compared to the SD group $(1.03 \pm 0.11)$. AdS-G increased scavenger receptor mRNA expression in VSMC in $\mathrm{SD}(0.43 \pm 0.15)$, $\mathrm{VD}(0.90 \pm 0.03)$ and $\mathrm{AD}(1.07 \pm 0.02)$ groups following a similar pattern but at a lesser extent than AdS-I. Finally, a cumulative impact was observed in AdS-IG stimulation in $\mathrm{SD}(1.99 \pm 0.14)$ and $\mathrm{AD}(2.67 \pm 0.02)$ groups, compared to AdS-I and AdS-G; an effect absent in the VD group $(1.68 \pm 0.21)$.

On the other hand, Oil-Red-O staining was done to relate FA incorporation after supernatant treatment to CD36 increased levels. In SD groups, AdS-U showed no increase of FA incorporation compared to untreated cells. In HFD groups, supernatants increased FA incorporation in VSMC when stimulated with AdS-U (Figure S2), with a more marked coloration in $\mathrm{AD}$ groups compared to VD groups.

\section{DISCUSSION}

The physiologic alterations of VSMC triggered by adipocyte conditioned media was recently addressed in humans [13]. The novelty of this study is the evaluation of the impact of adipocyte-conditioned media on VSMC in a model of obesity and T2D.

Adipocytes were isolated from abdominal adipose tissues of male mice at different stages of metabolic alterations [11] to condition VSMC culture medium. The protocol was set to reproduce a culture environment that mimics in vivo hyperglycemic and/or hyperinsulinic conditions. Adipocytes were stimulated with glucose, insulin or both. Their impact on VSMC was compared to unstimulated cells.

Obesity-linked adipocyte alterations modulate VSMC migration, an important cellular process in atherogenesis. We previously demonstrated that AdS decreases VSMC proliferation and correlates with adiponectin and leptin unbalance [12].

In the present study, we showed that VSMC migration was statistically correlated with adiponectin and leptin changes in AdS. Indeed, recent observations have shown that adiponectin deprivation results in neointima hyperplasia, while leptin promotes VSMC migration [14,15]. Equivalent numbers of cultured adipocytes are not transposed in the same adipokine secretion profile in all diet groups. A sharp decrease in adiponectin levels, coupled to hyper-leptinemia, was correlated with increased VSMC migration in HFD groups. Insulin \pm glucose treatments further increased the migratory potential of VSMC, possibly due to higher secreted levels of leptin in AdS. Decreased adiponectin levels also contribute to the increase VSMC migration potential, since this adipokine is known to inhibit the migratory process [16]. These modulations were also reflected by the arrangement of the $\alpha$-actin filaments, as reported by confocal microscopy. We have shown different stages of filament disorganizations and correlated them with an increased level of migration in AdS extracted from HFD mice groups. These disorders in the cytoskeleton are most probably due to increased actin dis-polymerization and re-polymerization events, given stimulation of the migratory mechanism $[17,18]$. Dot staining shown in $\mathrm{AD}$ groups relates to a higher level of soluble actin monomers needed to polymerize and form filaments $[18,19]$. This will lead to this snapshot showing the formation of actin filaments that will push the leading cell front forward and catalyzing VSMC migration $[18,20]$.

In accordance with the above-discussed hypothesis, released FA could impact glucose cell homeostasis and 
response to insulin. InsR gene expression levels in VSMC were decreased in the presence of insulin + glucose, compared to unstimulated VSMC. This level was increased when cells were stimulated with glucose alone, compared to insulin + glucose stimulated cells. In the presence of AdS treatment, InsR mRNA levels increased in AdS-I and AdS-G from AD groups compared to both SD and VD groups. Saturated FA present at high concentrations in $\mathrm{AD}$ is known to block insulin activation [21] and induce hyperleptinemia, which has been reported to impair insulin signaling pathways [22]. This was noticed in this study with the lack of insulin-induced adiponectin release in AdS in HFD groups compared to SD. To counteract the effect of reduced glucose uptake, VSMC increase their InsR expression levels. In contrast, VD improves lipogenesis and lowers FA in AdS through its high level of oleic acid, which has been documented to trigger insulin sensitivity [23] and lead to a lower InsR mRNA levels.

Adipocytes used in this protocol were conditioned by food diets during 20 weeks. Mice of HFD groups were hyperglycemic; a status usually associated with elevated advanced glycation end products (A-GEP). We hypothesized that A-GEP present in these adipocytes attenuate their insulin sensitivity and abolish insulin-activated lipogenesis [24]. In this situation, adipocytes from HFDgroups had increased lipolysis activity, which leads to FA release increase and VSMC migration [3,25].

These mice had an increased serum concentration of FA compared to SD; a profile directly linked to the activity of abdominal adipocytes. FA rich AdS from HFDmice can stimulate PPAR $\gamma$ in VSMC, triggering CD36 gene expression $[8,26]$. CD36 plays a key role in FA acid uptake and regulation in vivo [27].

Adipokines were also reported to regulate CD36 expression [28] and therefore FA accumulation in VSMC and morphologic modulations. While leptin increases CD36 expression in VSMC, adiponectin prevents it [29, 30]. Thus, VSMC treated with AdS from HFD groups modulate their CD36 expression in correlation with leptin and adiponectin levels contained in the culture media. CD36 mRNA upregulation levels are correlated with increased Oil-Red-O cell staining in corresponding diet groups, indicative of FA accumulation in VSMC cytoplasm [31]. This accumulation does not seem to influence migratory potentials, even though we have shown that AdS from HFD groups increases apoptotic factors [12]. In fact, it has been reported that caveolin contained in muscle cells sequester FA on the cytoplasmic membrane [32]. This could protect the actin fluency and migration potential even if the apoptosis process is triggered.

Further studies will be needed to further characterize this aspect of morphologic alteration and FA accumulation.
All together, these data indicate that the development of adipocyte-induced VSMC alterations is linked to the FA diet composition and the degree of metabolic alterations. Such alterations trigger adipokine unbalance from adipocytes, defining VSMC migration and FA cytoplasmic accumulation. Thus, adipocyte alterations would directly influence VSMC transmigration towards the arterial media and thereby inducing, at the same time, apoptosis and foam cell formation. Simultaneously, these events may trigger atherogenesis by the migration of VSMC-derived foam cells in the intima and stimulate inflammatory cell recruitment into the arterial wall. These results may contribute to better understand the complex interplay between adipocytes and vascular cells that lead to increased atherosclerotic events in obesity-linked metabolic syndromes.

\section{CONCLUSIONS AND LIMITATIONS}

The current study appears to be compelling, and makes a significant contribution to the field of atherogenesis process as well as the understanding of the impact of the adipose tissue on VSMC. It reflects the direct impact of adipose tissue secretion cocktail on the VSMC migratory potential; and it's also a main characteristic that affects atherogenesis. Further, the originality of this study is the evaluation of these secretions of adipocytes provided from mice groups showing different levels of metabolic alterations.

In spite of this, the current research acknowledges a few limitations that should be noted to help interpret the results, and be added to the data on the relationships between adipose tissue and vascular wall cells physiology. In fact, due to material limitations, we have used adipocytes extracted from abdominal white adipose tissue instead of those of perivascular adipose tissue that are in direct contact with the vascular wall. Even if these two types of tissue are comparable, their localization in the body could affect their physiology [33]. The amount of perivascular tissue is very limited in our protocol mice and the quantity couldn't cover all our stimulation conditions. However, we can speculate that the effect would be very close between these two types of white adipose tissues.

\section{ACKNOWLEDGMENTS}

The authors gratefully thank Louis Villeneuve from the Montreal Heart Institute for immunostaining and confocal microscopy advices and Mariève Cossette from the Montreal Heart Institute Coordinating Centre for the statistical analyses.

\section{REFERENCES}

[1] Casey, R.G., Gang, C., Joyce, M. and Bouchier-Hayes, 
D.J. (2007) Taurine attenuates acute hyperglycaemia-induced endothelial cell apoptosis, leucocyte-endothelial cell interactions and cardiac dysfunction. Journal of Vascular Research, 44, 31-39.

http://dx.doi.org/10.1159/000097893

[2] Hennig, B., Toborek, M. and McClain, C.J. (2001) Highenergy diets, fatty acids and endothelial cell function: Implications for atherosclerosis. Journal of the American College of Nutrition, 20, 97-105.

http://dx.doi.org/10.1080/07315724.2001.10719021

[3] Rodriguez-Lee, M., Ostergren-Lunden, G., Wallin, B., Moses, J., Bondjers, G. and Camejo, G. (2006) Fatty acids cause alterations of human arterial smooth muscle cell proteoglycans that increase the affinity for low-density lipoprotein. Arteriosclerosis, Thrombosis, and Vascular Biology, 26, 130-135.

http://dx.doi.org/10.1161/01.ATV.0000191659.94059.62

[4] Ross, R. (1999) Atherosclerosis-An inflammatory disease. New England Journal of Medicine, 340, 115-126. http://dx.doi.org/10.1056/NEJM199901143400207

[5] Mulvihill, E.R., Jaeger, J., Sengupta, R., Ruzzo, W.L., Reimer, C., Lukito, S., et al. (2004) Atherosclerotic plaque smooth muscle cells have a distinct phenotype. Arteriosclerosis, Thrombosis, and Vascular Biology, 24, 12831289.

http://dx.doi.org/10.1161/01.ATV.0000132401.12275.0c

[6] Bentzon, J.F., Weile, C., Sondergaard, C.S., Hindkjaer, J., Kassem, M. and Falk, E. (2006) Smooth muscle cells in atherosclerosis originate from the local vessel wall and not circulating progenitor cells in ApoE knockout mice. Arteriosclerosis, Thrombosis, and Vascular Biology, 26, 2696-2702.

http://dx.doi.org/10.1161/01.ATV.0000247243.48542.9d

[7] Rong, J.X., Shapiro, M., Trogan, E. and Fisher, E.A. (2003) Transdifferentiation of mouse aortic smooth muscle cells to a macrophage-like state after cholesterol loading. Proceedings of the National Academy of Sciences of the United States of America, 100, 13531-13536. http://dx.doi.org/10.1073/pnas.1735526100

[8] Lim, H.J., Lee, S., Lee, K.S., Park, J.H., Jang, Y., Lee, E.J., et al. (2006) PPARgamma activation induces CD36 expression and stimulates foam cell like changes in rVSMCs. Prostaglandins \& Other Lipid Mediators, 80, 165-174.

http://dx.doi.org/10.1016/j.prostaglandins.2006.06.006

[9] Antuna-Puente, B., Feve, B., Fellahi, S. and Bastard, J.P. (2008) Adipokines: The missing link between insulin resistance and obesity. Diabetes \& Metabolism, 34, 2-11. http://dx.doi.org/10.1016/j.diabet.2007.09.004

[10] Fantuzzi, G. (2005) Adipose tissue, adipokines, and inflammation. Journal of Allergy and Clinical Immunology, 115, 911-919. http://dx.doi.org/10.1016/i.jaci.2005.02.023

[11] El Akoum, S., Lamontagne, V., Cloutier, I. and Tanguay, J.F. (2011) Nature of fatty acids in high fat diets differentially delineates obesity-linked metabolic syndrome components in male and female C57BL/6J mice. Diabetology \& Metabolic Syndrome, 3, 34.

http://dx.doi.org/10.1186/1758-5996-3-34

[12] El Akoum, S., Cloutier, I. and Tanguay, J.F. (2011) Vas- cular smooth muscle cell alterations triggered by mice adipocytes: Role of high-fat diet. Journal of Atherosclerosis and Thrombosis, 19, 1128-1141. http://dx.doi.org/10.5551/jat.13482

[13] Lamers, D., Schlich, R., Greulich, S., Sasson, S., Sell, H. and Eckel, J. (2011) Oleic acid and adipokines synergize in inducing proliferation and inflammatory signaling in human vascular smooth muscle cells. Journal of Cellular and Molecular Medicine, 15, 1177-1188. http://dx.doi.org/10.1111/j.1582-4934.2010.01099.x

[14] Kubota, N., Terauchi, Y., Yamauchi, T., Kubota, T., Moroi, M., Matsui, J., et al. (2002) Disruption of adiponectin causes insulin resistance and neointimal formation. Journal of Biological Chemistry, 277, 25863-25866. http://dx.doi.org/10.1074/jbc.C200251200

[15] Oda, A., Taniguchi, T. and Yokoyama, M. (2001) Leptin stimulates rat aortic smooth muscle cell proliferation and migration. Kobe Journal of Medical Sciences, 47, 141150.

[16] Asterholm, I.W. and Scherer, P.E. (2010) Enhanced metabolic flexibility associated with elevated adiponectin levels. American Journal of Pathology, 176, 1364-1376. http://dx.doi.org/10.2353/ajpath.2010.090647

[17] Wanger, M. and Wegner, A. (1985) Equilibrium constant for binding of an actin filament capping protein to the barbed end of actin filaments. Biochemistry, 24, 10351040. http://dx.doi.org/10.1021/bi00325a035

[18] Pollard, T.D. and Borisy, G.G. (2003) Cellular motility driven by assembly and disassembly of actin filaments. Cell, 112, 453-465. http://dx.doi.org/10.1016/S0092-8674(03)00120-X

[19] Wang, Y.L. (1985) Exchange of actin subunits at the leading edge of living fibroblasts: Possible role of treadmilling. Journal of Cell Biology, 101, 597-602. http://dx.doi.org/10.1083/jcb.101.2.597

[20] Mitchison, T.J. and Cramer, L.P. (1996) Actin-based cell motility and cell locomotion. Cell, 84, 371-379. http://dx.doi.org/10.1016/S0092-8674(00)81281-7

[21] Chavez, J.A. and Summers, S.A. (2003) Characterizing the effects of saturated fatty acids on insulin signaling and ceramide and diacylglycerol accumulation in 3T3-L1 adipocytes and C2C12 myotubes. Archives of Biochemistry and Biophysics, 419, 101-109. http://dx.doi.org/10.1016/j.abb.2003.08.020

[22] Benomar, Y., Wetzler, S., Larue-Achagiotis, C., Djiane, J., Tome, D. and Taouis, M. (2005) In vivo leptin infusion impairs insulin and leptin signalling in liver and hypothalamus. Molecular and Cellular Endocrinology, 242, 59-66. http://dx.doi.org/10.1016/j.mce.2005.07.003

[23] Soriguer, F., Esteva, I., Rojo-Martinez, G., Ruiz de Adana, M.S., Dobarganes, M.C., Garcia-Almeida, J.M., et al. (2004) Oleic acid from cooking oils is associated with lower insulin resistance in the general population (Pizarra study). European Journal of Endocrinology, 150, 33-39. http://dx.doi.org/10.1530/eje.0.1500033

[24] Unoki, H., Bujo, H., Yamagishi, S., Takeuchi, M., Imaizumi, T. and Saito Y. (2007) Advanced glycation end products attenuate cellular insulin sensitivity by increasing the generation of intracellular reactive oxygen species in 
adipocytes. Diabetes Research and Clinical Practice, 76, 236-244. http://dx.doi.org/10.1016/j.diabres.2006.09.016

[25] Artwohl, M., Lindenmair, A., Roden, M., Waldhausl, W.K., Freudenthaler, A., Klosner, G., et al. (2009) Fatty acids induce apoptosis in human smooth muscle cells depending on chain length, saturation, and duration of exposure. Atherosclerosis, 202, 351-362. http://dx.doi.org/10.1016/j.atherosclerosis.2008.05.030

[26] Sharma, A.M. and Staels, B. (2007) Review: Peroxisome proliferator-activated receptor gamma and adipose tissue-Understanding obesity-related changes in regulation of lipid and glucose metabolism. Journal of Clinical Endocrinology \& Metabolism, 92, 386-395. http://dx.doi.org/10.1210/jc.2006-1268

[27] Febbraio, M., Abumrad, N.A., Hajjar, D.P., Sharma, K., Cheng, W., Pearce, S.F., et al. (1999) A null mutation in murine CD36 reveals an important role in fatty acid and lipoprotein metabolism. Journal of Biological Chemistry, 274, 19055-19062. http://dx.doi.org/10.1074/jbc.274.27.19055

[28] Lee, T.S., Lin, C.Y., Tsai, J.Y., Wu, Y.L., Su, K.H., Lu, K.Y., et al. (2009) Resistin increases lipid accumulation by affecting class A scavenger receptor, CD36 and ATPbinding cassette transporter-A1 in macrophages. Life Sciences, 84, 97-104.

http://dx.doi.org/10.1016/j.lfs.2008.11.004

[29] Konstantinidis, D., Paletas, K., Koliakos, G. and Kaloyianni, M. (2008) Signaling components involved in leptin-induced amplification of the atherosclerosis-related

\section{SUPPLEMENTARY}

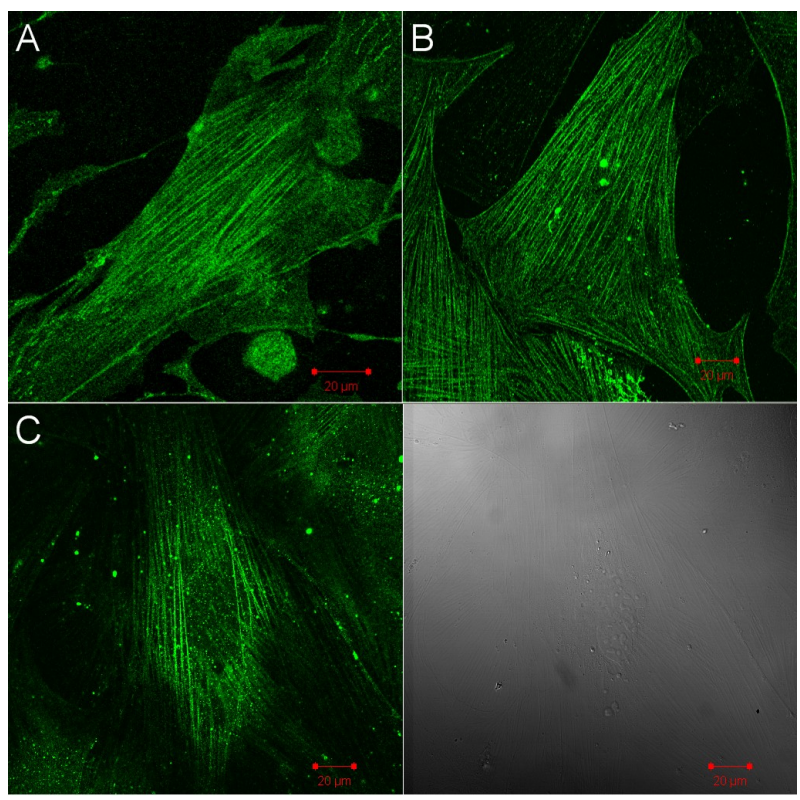

Figure S1. Immunocytochemical characterization of VSMC as observed on confocal microscopy after a $24 \mathrm{~h}$ treatment with AdS from SD (A); VD (B) or AD (C) groups. $\alpha$-actin filament disorganization level was increased depending on the diet nature group. properties of human monocytes. Journal of Vascular Research, 46, 199-208. http://dx.doi.org/10.1159/000161234

[30] Ouchi, N., Kihara, S., Arita, Y., Nishida, M., Matsuyama, A., Okamoto, Y., et al. (2001) Adipocyte-derived plasma protein, adiponectin, suppresses lipid accumulation and class A scavenger receptor expression in human monocyte-derived macrophages. Circulation, 103, 1057-1063. http://dx.doi.org/10.1161/01.CIR.103.8.1057

[31] Sanchez-Hidalgo, M., Lu, Z., Tan, D.X., Maldonado, M.D., Reiter, R.J. and Gregerman, R.I. (2007) Melatonin inhibits fatty acid-induced triglyceride accumulation in ROS17/2.8 cells: Implications for osteoblast differentiation and osteoporosis. American Journal of Physiology. Regulatory, Integrative and Comparative Physiology, 292, R2208-R2215. http://dx.doi.org/10.1152/ajpregu.00013.2007

[32] Simard, J.R., Meshulam, T., Pillai, B.K., Kirber, M.T., Brunaldi, K., Xu, S., et al. (2010) Caveolins sequester FA on the cytoplasmic leaflet of the plasma membrane, augment triglyceride formation, and protect cells from lipotoxicity. Journal of Lipid Research, 51, 914-922. http://dx.doi.org/10.1194/jlr.M900251

[33] Palou, M., Sanchez, J., Priego, T., Rodriguez, A.M., Pico, C. and Palou, A. (2010) Regional differences in the expression of genes involved in lipid metabolism in adipose tissue in response to short- and medium-term fasting and refeeding. Journal of Nutritional Biochemistry, 21, 23-33. http://dx.doi.org/10.1016/j.jnutbio.2008.10.001

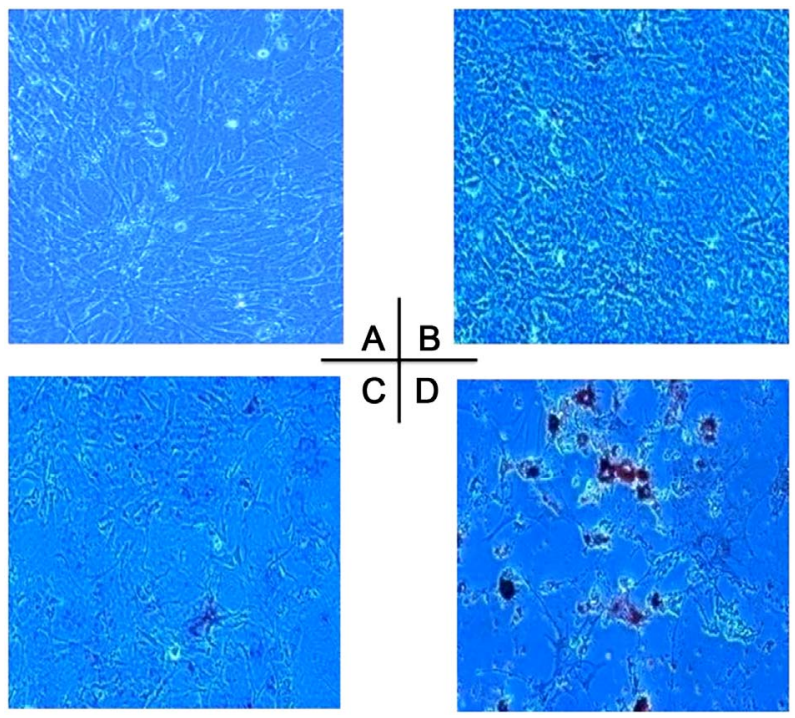

Figure S2. FA accumulation reflected by Oil Red O staining in native VSMC (A) and in VSMC after a $24 \mathrm{~h}$ treatment with AdS from SD (B); VD (C) or AD (D) groups. FA accumulation was increased with AdS treatment at different level depending on the diet nature group. 